\title{
Physician Survey on Cancer Susceptibility Testing
}

National Cancer Institute

\section{Source}

National Cancer Institute. Physician Survey on Cancer Susceptibility Testing. NCI

Thesaurus. Code C20091.

The objectives of this survey are to: 1 ) determine the utilization of genetic tests by physicians at the national level, 2) ascertain physician knowledge of available genetic tests for specific cancer susceptibility genes, 3) examine physicians' general attitudes toward testing, 4) explore possible variation in utilization and knowledge/attitudes by medical specialty, type of practice, year of training completion, board status, urbanization, and geographic region. 